\title{
Boundary and Medial Shape Analysis of the Hippocampus in Schizophrenia
}

\author{
Martin Styner ${ }^{1}$, Jeffrey A. Lieberman ${ }^{2}$, and Guido Gerig ${ }^{2,3}$ \\ 1 M.E. Müller Research Center for Orthopaedic Surgery, Institute for Surgical \\ Technology and Biomechanics, University of Bern, P.O. Box 8354, 3001 Bern \\ martin_styner@ieee.org \\ 2 Department of Psychiatry, University of North Carolina at Chapel Hill, CB\#7160, \\ Chapel Hill, NC 27599-7160 \\ 3 Department of Computer Science, University of North Carolina at Chapel Hill, \\ CB\#3175, Sitterson Hall, Chapel Hill, NC 27599-3175 ${ }^{\dagger}$
}

\begin{abstract}
Statistical shape analysis has become of increasing interest to the neuroimaging community due to its potential to precisely locate morphological changes and thus potentially discriminate between healthy and pathological structures. This paper describes a combined boundary and medial shape analysis based on two different shape descriptions applied to a study of the hippocampus shape abnormalities in schizophrenia. The first shape description is the sampled boundary implied by the spherical harmonic SPHARM description. The second one is the medial shape description called M-rep. Both descriptions are sampled descriptions with inherent point correspondence. Their shape analysis is based on computing differences from an average template structure analyzed using standard group mean difference tests. The results of the global and local shape analysis in the presented hippocampus study exhibit the same patterns for the boundary and the medial analysis. The results strongly suggest that the normalized hippocampal shape of the schizophrenic group is different from the control group, most significantly as a deformation difference in the tail region.
\end{abstract}

\section{Introduction}

Quantitative morphologic assessment of individual brain structures is often based on volumetric measurements. Volume changes are intuitive features as they might explain atrophy or dilation due to illness. On the other hand, structural changes at specific locations are not sufficiently reflected in global volume measurements. Shape analysis has thus become of increasing interest to the neuroimaging community due to its potential to precisely locate morphological changes.

\footnotetext{
${ }^{\dagger}$ We are thankful to Christian Brechbühler for providing the SPHARM software, to Steve Pizer and Sarang Joshi of the UNC MIDAG group for providing M-rep tools, to Scott Schobel for segmenting the hippocampi and to Maya Styner for editorial assistance. The hippocampal schizophrenia study was funded by the Stanley Foundation. This work was also funded by NCI grant CA 47982 and NIMH grant P30-MH33127.
} 
One of the first and most influential research in shape analysis was presented by D'Arcy Thomsonw [1] in his ground-breaking book On Growth and Form. In more recent years, several researchers proposed shape analysis via deformable registration to a template 2345 . Inter-subject comparisons are made by analyzing the individual deformable transformations. This analysis of the transformation fields has to cope with the high dimensionality of the transformation, the template selection problem and the sensitivity to the initial position. Nevertheless, several studies have shown stable shape analysis results. Bookstein et al. 6] and Dryden et al. 7] presented some of the first mathematical methods for 3D shape analysis based on sampled descriptions. The shape analysis of densely sampled 3D Point Distribution Models (PDM) and their deformations was first investigated by Cootes and Taylor [8]. Inspired by their experiments, Gerig et al. 9] proposed shape analysis based on a parametric boundary description called SPHARM [10. The SPHARM shape analysis approach was extended by Styner et al. to use the implied PDM [11], a method recently also used by Shen et al. 12. Pizer et al.1314 and Golland et al. 15] proposed shape analysis on medial shape descriptions in $3 \mathrm{D}$ and $2 \mathrm{D}$, repectively. They used a fixed topology sampled model with implicit correspondence that is fitted to the objects.

In this paper we present the comparison of a sampled boundary representation (PDM derived from SPHARM) and a sampled medial description (M-rep) which leads to discussions of their strengths and limitations. In the next section, these methods are described and in the result section, a shape study of the hippocampus structure in the setting of schizophrenia is presented.

\section{Methods}

This section first describes the description of objects using the SPHARM shape description, followed by the template based shape analysis. Next, the M-rep description and its shape analysis method are described. Alignment and scaling of the objects are two important issues in shape analysis that are not discussed in detail here (see [11]). For both SPHARM and M-rep, the objects are normalized prior to the shape analysis by rigid-body Procrustes alignment [16] and by scaling to unit volume. We chose volume scaling since many clinical studies with different anatomical objects provided optimal shape discrimination using this normalization scheme.

\subsection{Boundary Shape Analysis via SPHARM}

The SPHARM description is a parametric boundary description that can only represent objects of spherical topology [10]. The spherical parameterization is computed via optimizing an equal area mapping of the $3 \mathrm{D}$ voxel mesh onto the sphere and minimizing angular distortions. The basis functions of the parameterized boundary are spherical harmonics. Each individual SPHARM description is composed of a set of coefficients, weighting the basis functions. Based on a uniform icosahedron-subdivision of the spherical parameterization, we obtain a 


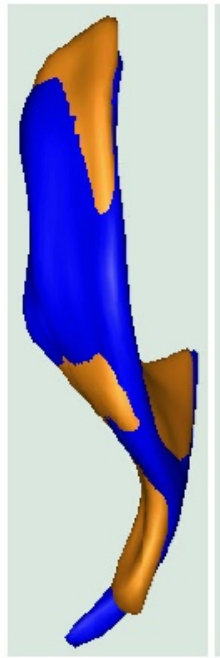

A

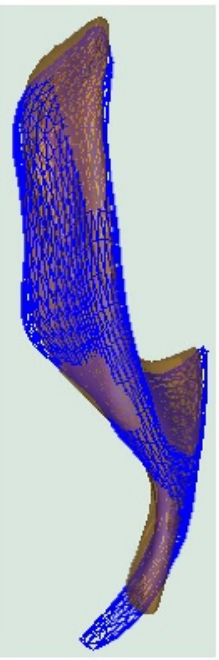

B

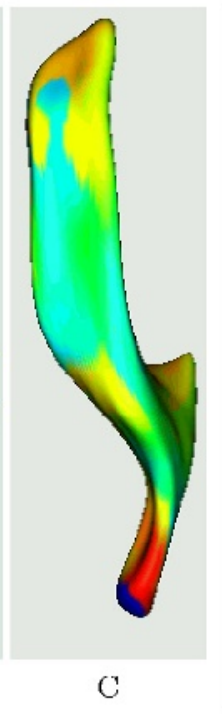

$\mathrm{C}$
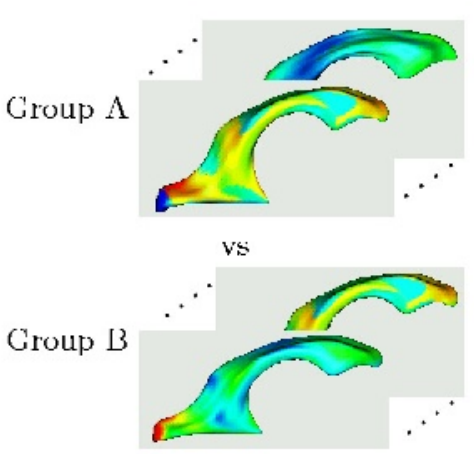

$\downarrow$

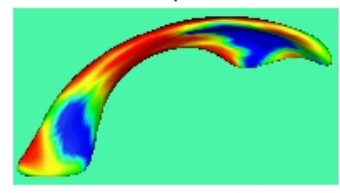

$8 \mathrm{~mm}$

Fig. 1. SPHARM shape analysis. Left: Signed distance map computation between an individual object (blue) and a template structure (orange). A: Objects after alignment and scaling. B: Same as A, but the template is shown transparent and the object as grid-mesh. C: Distance map with color-coded distance at each boundary-point. Right: Statistical map computation: For two groups of objects, distance maps are compared in statistical tests yielding a statistical map. The significance map shows the color coded significance.

Point Distribution Model (PDM) 17. Correspondence of SPHARM is determined by normalizing the parameterization to the first order ellipsoid [1].

The SPHARM shape analysis briefly discussed here is visualized in Figure 1 using a lateral ventricle structure (more detailed in [11]). The basis of the analysis as discussed here is the SPHARM implied PDM. Prior to the shape analysis, the group average object is computed for each subject group, and an overall average object is computed over all group average objects. This overall average object is used in the shape analysis as the template object.

At every PDM boundary point for each SPHARM object, we compute a distance map representing the signed local Euclidean surface distance to the template object. The sign of the local distance is computed using the direction of the template surface normal. In the global shape analysis, the mean of the local distances is analyzed with a standard group mean difference test. The local shape analysis is computed by testing the local distances at every boundary point. This results in a significance map that represents the significance of these local statistical tests and thus allows locating significant shape differences between the groups. The local shape analysis is not corrected for multiple, correlated statistical tests and thus the significance map is an optimistic estimate. 


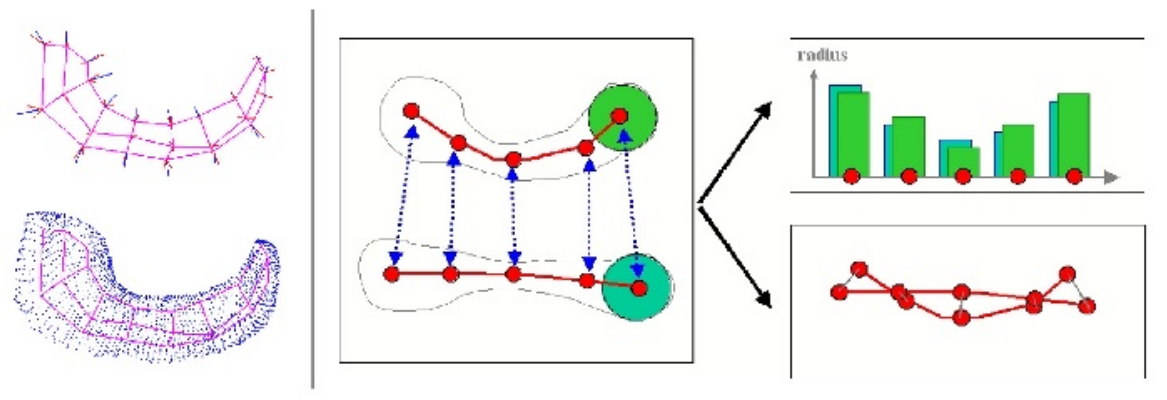

Fig. 2. Left: Single figure M-rep of a hippocampus without (top) and with (bottom) implied boundary from superior view. Right: M-rep shape difference (schematically in 2D) of 2 medial objects: Differences in the thickness (top graph) and position (lower graph) are studied separately. The properties express different kinds of underlying processes (growth vs. deformation).

\subsection{Medial Shape Analysis via M-Rep}

An M-rep ([13]) is a linked set of medial primitives called medial atoms, $m=(x, r, \underline{F}, \theta)$. The atoms are formed from two equal length vectors and are composed of 1) a position $x, 2)$ a radius $r, 3)$ a frame $\underline{F}$ implying the tangent plane to the medial manifold and 4) an object angle $\theta$. The medial atoms are grouped by intra-figural links into figures that are connected by inter-figural links. Via interpolation, a fully connected boundary is implied by the M-rep. The single figure M-rep of a hippocampus object is visualized in Figure 2 with its implied boundary. The individual M-rep description is determined by fitting a previously computed M-rep model to the object-boundary. The model generation and the fitting process are described in [18].

In contrast to the boundary shape analysis, a medial shape analysis separately analyzes the two medial shape properties: local position and thickness 14 . This separation provides additional statistical power to the analysis. Figure 2 demonstrates how thickness and position capture different forms of shape deformation, i.e. thickness changes are due to locally uniform growth forces and positional changes are due to local deformation forces. Since the M-rep model is based on a coarse grid of medial atoms, the M-rep shape analysis captures only large scale shape differences, whereas the SPHARM shape analysis captures both small and large scale shape differences.

In the medial shape analysis, an approach similar to the SPHARM shape analysis is applied. First the M-rep description of the overall average object is computed as the template. Then, the signed position and thickness differences to the template are computed for each M-rep. The sign of the position difference is computed using the direction of the template medial surface normals. In the global shape analysis, the mean of the local differences is analyzed by standard mean difference tests. The local shape analysis is computed by testing each medial atom independently. Although this local shape analysis is not corrected for 
Table 1. Global shape analysis: Table of group mean difference $p$-values between the schizophrenic and control group (ns = non-significant at $5 \%$ significance level).

\begin{tabular}{l|r|r|r} 
Global SZ vs. Cnt & SPHARM Distance & M-rep Thickness & M-rep Position \\
\hline Left & (ns) $p=0.154$ & (ns) $p=0.722$ & (ns) $p=0.0513$ \\
Right & $p=0.015$ & (ns) $p=0.751$ & $p=0.0001$
\end{tabular}

multiple, correlated statistical tests, the computed local statistics is appropriate for a preliminary quantitative shape analysis due to low number of medial atoms.

\section{$3 \quad$ Results}

We investigated the shape of the hippocampus structure in schizophrenic patients (SZ, 56 cases) and healthy controls (Cnt, 26 cases) of male gender and same handedness, as well as matched for age and ethnicity. The goal of this study was to assess shape changes between the patient and control groups. The hippocampi were segmented from IRprepped SPGR MRI datasets (0.9375x0.9375x1.5mm) using a manual outlining procedure based on a strict protocol and well-accepted anatomical landmarks ([19]). The segmentation was performed by a single clinical expert (20]) with intra-rater variability of the segmented volume measurements at 0.95 . The SPHARM coefficients were computed from the segmentation. The SPHARM implied PDM's were computed using a sampling of 2252 points along the boundary. The M-rep model was then computed on the full SPHARM population. The resulting M-rep model has a single figure topology and a grid sampling of 3 by 8 atoms, in total 24 medial atoms. The objects were normalized as described in section 2 .

The template for both boundary and medial shape analysis was determined by the overall average structure. Due to variation of the patient population in regard to age and ethnicity, the shape difference values were corrected for age and influence (linear least square model). The shape analysis was computed for the uncorrected and corrected values, exhibiting very similar patterns. In this paper only the corrected analysis is presented.

The global shape analysis in Table 1 shows that only the right hippocampus is significantly differently shaped at the $5 \%$ significance level in the SPHARM analysis and the M-rep position analysis. A strong trend in the M-rep position analysis is also visible on the left side. The M-rep thickness analysis is neither significant for the left nor for the right hippocampus. This suggest a deformation shape change in the hippocampus between the schizophrenic and the control group. The results of the M-rep position analysis shows a stronger significance than the SPHARM analysis. Additionally to the mean difference, several quartile measures were analyzed and produced structurally the same results.

The local analysis visualized in significance maps (see Fig. 3) shows a similar pattern of regions of significant difference in the SPHARM shape analysis as in the M-rep position shape analysis. No significance was found in the M-rep thickness analysis. Similar to the outcome of the global analysis, the local M-rep 

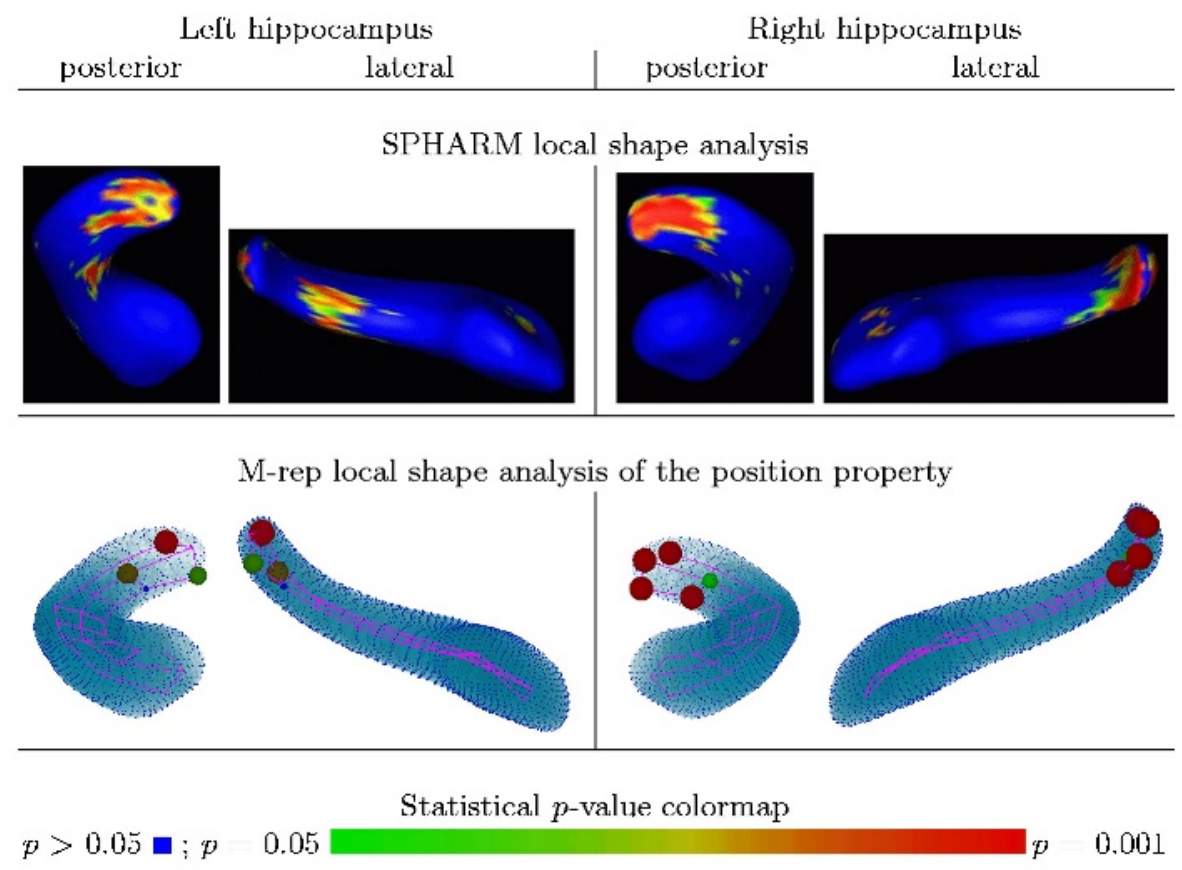

Fig. 3. Statistical maps of the local shape analysis from posterior and lateral views. Top row: SPHARM shape analysis, bottom row: M-rep shape analysis of the position property. The M-rep shape analysis of thickness property is not shown since no regions of significance are present. The M-rep analysis shows the statistical significance at each medial atom using both the color and the radius of spheres placed at the atom positions. The patterns of the local analysis are similar for both SPHARM and M-rep analysis. The main area of significance is located at the hippocampal tail.

position analysis shows a stronger significance than the SPHARM analysis. The local shape differences are mainly located at the hippocampal tail on both the left and the right side. Thus, this local shape analysis suggests a deformation shape difference between the schizophrenic and control group located at the hippocampal tail. By inspecting the average structures of the two groups, we further find that the hippocampus of the control groups is more bent than the one of the schizophrenic group.

\section{Discussion and Conclusions}

We have presented a comparison of the boundary SPHARM and medial Mrep shape analysis for both global and local changes. The analysis uses similar statistical methods for both the medial and the boundary description, but the descriptions themselves are fundamentally different. The results show a good 
concordance between the detected changes in the SPHARM and the M-rep analysis. This concordance strengthens the validity of the reported results.

In the presented study, the M-rep position shape analysis is statistically more significant for both the global and local statistics than the SPHARM analysis. This is mainly due to separation of medial properties of thickness and position, since the thickness information seems to contain no relevant information and thus effectively additional noise is present in the SPHARM shape analysis. The M-rep analysis here also provides the additional information of the presence of deformation change and the absence of growth or atrophy. This information cannot be deducted from the SPHARM analysis. Also the low number of medial atoms, 24 atoms in the presented study, allows a more appropriate estimation of the local statistics. The local SPHARM analysis is likely to produce an overly optimistic estimation as well as false-positives.

The main results of the shape analysis study is the presence of significant hippocampal abnormalities in the schizophrenia patients. The pattern of shape abnormality clearly shows a hippocampal shape change in the tail region due to deformation. This is an interesting result as it suggests deformation of the hippocampal tail at a position where it connects to the fimbria. Future shape analysis of objects in the context of embedded objects will help to explain the the reason for such a finding. In contrast to these results, Csernansky et al. [5] reported local shape analysis results of hippocampal abnormalities in schizophrenia located mainly in the head region, but also, to a minor extent, in the tail. Their shape analysis method is very different from ours and is based on the analysis of a high dimensional brain mapping procedure. It is yet unclear to us whether the source of the divergence of the results is the differences between the methods or the differences between the studied populations. An ongoing study at UNC currently applies the high dimensional warping method to the same study. This will result in a unique sample set that has the potential to decouple methodological differences from population differences.

The presented local shape analysis for both SPHARM and M-rep description is not corrected for multiple, correlated statistical tests. Thus the quantitative aspects of the local shape analysis results should be regarded as being preliminary. The development of a correction scheme is part of our ongoing research.

The combined SPHARM and M-rep shape analysis scheme is currently applied to other brain structures in schizophrenia and normal brain development studies. These studies show preliminary results with similarly good concordance between SPHARM and M-rep shape analysis.

\section{References}

1. D. Thomson, On Growth and Form, Cambridge University Press, second edition, 1942.

2. C. Davatzikos, M. Vaillant, S. Resnick, J.L Prince, S. Letovsky, and R.N. Bryan, "A computerized method for morphological analysis of the corpus callosum," Journal of Computer Assisted Tomography, vol. 20, pp. 88-97, Jan./Feb 1996.

3. S. Joshi, M Miller, and U. Grenander, "On the geometry and shape of brain sub-manifolds," Pat. Rec. Art. Intel., vol. 11, pp. 1317-1343, 1997. 
4. J.G. Csernansky, S. Joshi, L.E. Wang, J. Haller, M. Gado, J.P. Miller, U. Grenander, and M.I. Miller, "Hippocampal morphometry in schizophrenia via high dimensional brain mapping," Proc. Natl. Acad. Sci. USA, vol. 95, pp. 11406-11411, September 1998.

5. J.G. Csernansky, D. J. Wang, L. andd Jones, D. Rastogi-Cru, G. Posener, J.A.and Heydebrand, J.P. Miller, U. Grenander, and M.I. Miller, "Hippocampal deformities in schizophrenia characterized by high dimensional brain mapping," Am. J. Psychiatry, vol. 159, pp. 1-7, December 2002.

6. F.L. Bookstein, "Shape and the Information in Medical Images: A Decade of the Morphometric Synthesis," Comp. Vision and Image Under., vol. 66, no. 2, pp. 97-118, May 1997.

7. I.L. Dryden and K.V. Mardia, "Multivariate shape analysis," Sankhya, vol. 55, pp. 460-480, 1993.

8. T. Cootes, C.J. Taylor, D.H. Cooper, and J. Graham, "Active shape models their training and application," Computer Vision and Image Understanding, vol. 61, pp. 38-59, 1995.

9. G. Gerig, M. Styner, M.E. Shenton, and J.A. Lieberman, "Shape versus size: Improved understanding of the morphology of brain structures," in MICCAI, 2001, pp. 24-32.

10. C. Brechbühler, G. Gerig, and O. Kübler, "Parametrization of closed surfaces for 3-D shape description," Computer Vision, Graphics, Image Processing: Image Understanding, vol. 61, pp. 154-170, 1995.

11. G. Gerig, M. Styner, D. Jones, D. Weinberger, and J. Lieberman, "Shape analysis of brain ventricles using spharm," in MMBIA. 2001, pp. 171-178, IEEE press.

12. L. Shen, J. Ford, F. Makedon, and A. Saykin, "Hippocampal shape analysis surfacebased representation and classification," in SPIE-Medical Imaging, 2003.

13. S. Pizer, D. Fritsch, P. Yushkevich, V. Johnson, and E. Chaney, "Segmentation, registration, and measurement of shape variation via image object shape," IEEE Trans. Med. Imaging, vol. 18, pp. 851-865, Oct. 1999.

14. M. Styner, G. Gerig, J. Lieberman, D. Jones, and D. Weinberger, "Statistical shape analysis of neuroanatomical structures based on medial models," Medical Image Analysis, 2003, to appear.

15. P. Golland, W.E.L. Grimson, and R. Kikinis, "Statistical shape analysis using fixed topology skeletons: Corpus callosum study," in Information Processing in Medical Imaging, 1999, pp. 382-388.

16. F.L. Bookstein, Morphometric Tools for Landmark Data: Geometry and Biology, Cambridge University Press, 1991.

17. A. Kelemen, G. Székely, and G. Gerig, "Elastic model-based segmentation of 3d neuroradiological data sets," IEEE Trans. Med. Imaging, vol. 18, pp. 828-839, October 1999.

18. M Styner and G. Gerig, "Automatic and robust computation of 3d medial models incorporating object variability," Int. J. Comp. Vision, 2003, to be published.

19. H. M. Duvernoy, The human hippocampus, Functional Anatomy, Vascularization and serial Sections with MRI, Springer, 1998.

20. S Schobel, M. Chakos, G. Gerig, H. Bridges, H. Gu, H.C. Charles, and J. Lieberman, "Duration and severity of illness and hippocampal volume in schizophrenia as assessed by 3d-manual segmentation," Schizophrenia Research, vol. 49, no. 1-2, pp. 165, 2001. 